\title{
On Addition Formulae of KP, mKP and BKP Hierarchies
}

Yoko SHIGYO

Department of Mathematics, Tsuda College, Kodaira, Tokyo, 187-8577, Japan

E-mail: yoko.shigyo@gmail.com

Received December 12, 2012, in final form April 04, 2013; Published online April 23, 2013

http://dx.doi.org/10.3842/SIGMA.2013.035

\begin{abstract}
In this paper we study the addition formulae of the KP, the mKP and the BKP hierarchies. We prove that the total hierarchies are equivalent to the simplest equations of their addition formulae. In the case of the KP and the mKP hierarchies those results had previously been proved by Noumi, Takasaki and Takebe by way of wave functions. Here we give alternative and direct proofs for the case of the KP and mKP hierarchies. Our method can equally be applied to the BKP hierarchy.
\end{abstract}

Key words: KP hierarchy; modified KP hierarchy; BKP hierarchy

2010 Mathematics Subject Classification: 14H70; 37K10; 37K20

\section{Introduction}

Our purpose is to prove that some integrable hierarchies are equivalent to the simplest equations of their addition formulae. In this paper we study the KP, the modified KP (mKP) and the BKP hierarchies.

The (bilinear) KP hierarchy [12] is an infinite system of bilinear equations for $\tau(x), x=$ $\left(x_{1}, x_{2}, \ldots\right)$, given, in the generating form, by

$$
\oint e^{-2 \xi(y, \lambda)} \tau\left(x-y-\left[\lambda^{-1}\right]\right) \tau\left(x+y+\left[\lambda^{-1}\right]\right) \frac{d \lambda}{2 \pi i}=0 .
$$

Namely, if we expand the left hand side in $y=\left(y_{1}, y_{2}, \ldots\right)$, then we get Hirota's bilinear equations, which contain, as the simplest equation, the Kadomtsev-Petviashvili (KP) equation in the bilinear form

$$
\left(D_{1}^{4}+3 D_{2}^{2}-4 D_{1} D_{3}\right) \tau \cdot \tau=0
$$

where $D_{i}$ is the Hirota's bilinear operator defined by

$$
\left(D_{1}^{i_{1}} D_{2}^{i_{2}} \cdots\right) \tau \cdot \tau=\left.\left(\left(\frac{\partial}{\partial y_{1}}\right)^{i_{1}}\left(\frac{\partial}{\partial y_{2}}\right)^{i_{2}} \cdots\right)(\tau(x+y) \tau(x-y))\right|_{y=0}, \quad y=\left(y_{1}, y_{2}, \ldots\right) .
$$

If we put $y=\left(\sum_{i=1}^{m-1}\left[\beta_{i}\right]-\sum_{i=1}^{m+1}\left[\alpha_{i}\right]\right) / 2,[\alpha]=\left(\alpha, \alpha^{2} / 2, \alpha^{3} / 3, \ldots\right)$, instead of expanding in $y$, and compute the integral by taking residues, then we get addition formulae [20]:

$$
\sum_{i=1}^{m+1}(-1)^{i-1} C_{\alpha \beta} \tau\left(x+\sum_{j=1}^{m-1}\left[\beta_{j}\right]+\left[\alpha_{i}\right]\right) \tau\left(x+\sum_{j=1, j \neq i}^{m+1}\left[\alpha_{j}\right]\right)=0, \quad m \geq 2,
$$


where $C_{\alpha \beta}$ depend only on $\left\{\alpha_{i}\right\}_{1 \leq i \leq m+1}$ and $\left\{\beta_{i}\right\}_{1 \leq i \leq m-1}$. The simplest case of addition formulae is the case of $m=2$ :

$$
\begin{gathered}
\alpha_{12} \alpha_{34} \tau\left(x+\left[\alpha_{1}\right]+\left[\alpha_{2}\right]\right) \tau\left(x+\left[\alpha_{3}\right]+\left[\alpha_{4}\right]\right)-\alpha_{13} \alpha_{24} \tau\left(x+\left[\alpha_{1}\right]+\left[\alpha_{3}\right]\right) \tau\left(x+\left[\alpha_{2}\right]+\left[\alpha_{4}\right]\right) \\
+\alpha_{14} \alpha_{23} \tau\left(x+\left[\alpha_{1}\right]+\left[\alpha_{4}\right]\right) \tau\left(x+\left[\alpha_{2}\right]+\left[\alpha_{3}\right]\right)=0
\end{gathered}
$$

where $\alpha_{i j}=\alpha_{i}-\alpha_{j}$. It is surprising that the KP hierarchy itself is equivalent to (2). This fact has been proved by Takasaki and Takebe [23] by way of the wave functions of the KP hierarchy. Here we give an alternative and direct proof. First we show that the totality of addition formulae (1) is equivalent to the KP hierarchy by using the properties of symmetric functions. If we call the function of the form $\tau\left(x+\left[\alpha_{1}\right]+\cdots+\left[\alpha_{n}\right]\right)$ the $n$-point function, then (2) is a relation among two point functions. By shifting $x$ appropriately we can consider (2) as an expression of a four point function in terms of two point functions (see Proposition 2). Repeating this process, we can derive the formulae which express the $2 m$-point function as a determinant of 2-point functions. These formulae are called Fay's determinant formulae in the case of theta function [8, 19]. In [8] it is indicated without proofs that the determinant formulae can be obtained from the trisecant formulae corresponding to (2). In this sense the determinant formulae (11) and their derivation from (2) cannot be considered a new result ${ }^{1}$. Next we show that the Plücker's relations for the determinants appearing in these formulae are nothing but the addition formulae (1) for $m$-point functions. In this way, we can prove that (2) is equivalent to the KP hierarchy. For the mKP and the BKP hierarchies, similar results hold although there are some differences.

The mKP hierarchy [12] is an infinite system of differential equations for an infinite number of functions $\tau_{l}(x), l \in \mathbb{Z}$. In this case there are an infinite number of the simplest addition formulae:

$$
\begin{aligned}
& \alpha_{23} \tau_{l}\left(x+\left[\alpha_{1}\right]\right) \tau_{l+1}\left(x+\left[\alpha_{2}\right]+\left[\alpha_{3}\right]\right)-\alpha_{13} \tau_{l}\left(x+\left[\alpha_{2}\right]\right) \tau_{l+1}\left(x+\left[\alpha_{1}\right]+\left[\alpha_{3}\right]\right) \\
& +\alpha_{12} \tau_{l}\left(x+\left[\alpha_{3}\right]\right) \tau_{l+1}\left(x+\left[\alpha_{1}\right]+\left[\alpha_{2}\right]\right)=0, \quad l \in \mathbb{Z} .
\end{aligned}
$$

It had been proved that (3) is equivalent to the mKP hierarchy in [17]. Here we prove the equivalence in a similar strategy to the case of KP. A new feature of the present case is that there exist addition formulae involving $\tau_{l}$ and $\tau_{l+k}$ for $k \geq 2$. We prove that these addition formulae are consequences of (3).

The BKP hierarchy is an infinite system of bilinear equations for $\tau(x), x=\left(x_{1}, x_{3}, \ldots\right)$. The following is the simplest addition formula which has four terms:

$$
\begin{aligned}
& \tilde{\alpha}_{12} \tilde{\alpha}_{13} \alpha_{23} \tau\left(x+2\left[\alpha_{1}\right]_{\mathrm{o}}\right) \tau\left(x+2\left[\alpha_{2}\right]_{\mathrm{o}}+2\left[\alpha_{3}\right]_{\mathrm{o}}\right) \\
& \quad-\tilde{\alpha}_{12} \tilde{\alpha}_{23} \alpha_{13} \tau\left(x+2\left[\alpha_{2}\right]_{\mathrm{o}}\right) \tau\left(x+2\left[\alpha_{1}\right]_{\mathrm{o}}+2\left[\alpha_{3}\right]_{\mathrm{o}}\right) \\
& \quad+\tilde{\alpha}_{13} \tilde{\alpha}_{23} \alpha_{12} \tau\left(x+2\left[\alpha_{3}\right]_{\mathrm{o}}\right) \tau\left(x+2\left[\alpha_{1}\right]_{\mathrm{o}}+2\left[\alpha_{2}\right]_{\mathrm{o}}\right) \\
& \quad-\alpha_{12} \alpha_{13} \alpha_{23} \tau(x) \tau\left(x+2\left[\alpha_{1}\right]_{\mathrm{o}}+2\left[\alpha_{2}\right]_{\mathrm{o}}+2\left[\alpha_{3}\right]_{\mathrm{o}}\right)=0
\end{aligned}
$$

where $\tilde{\alpha}_{i j}=\alpha_{i}+\alpha_{j}$ and $[\alpha]_{\mathrm{o}}=\left(\alpha, \alpha^{3} / 3, \alpha^{5} / 5, \ldots\right)$. We prove that (4) is equivalent to the $\mathrm{BKP}$ hierarchy in a similar way to the KP hierarchy. In this case we use Pfaffians instead of determinants to express $n$-point functions in terms of one and two point functions. To this end we need the analogue of Sylvester's theorem and the Plücker's relations for Pfaffians [9, 18].

We have shown that the KP, the mKP and the BKP hierarchies are equivalent to the simplest addition formulae. It is interesting to study whether, for other integrable hierarchies $[3,4,5$, 12, 21, 22], similar structure exists. There exists a result for the Toda hierarchy [21, 24]. But the problem arises to specify what are the fundamental equations in general. To consider these problems, it will be effective to use free fermion descriptions of integrable hierarchies $[3,4,5,12]$.

\footnotetext{
${ }^{1}$ Takasaki K., Private communications.
} 
It is also interesting to apply the results to the study of discrete differential geometries $[1,11]$ and addition formulae for sigma functions $[2,7,16]$.

This paper consists of three sections and three appendices. In Section 2, we consider the KP hierarchy. The key point is to prove the equivalence between the KP hierarchy and its infinite sequence of addition formulae. Since this case is fundamental, the details are given. Then we consider the mKP hierarchy in Section 3. The arguments which are similar to the KP hierarchy are omitted. In Section 4, we study the BKP hierarchy. The Pfaffians are necessary in this case. Necessary properties of Pfaffians including the definition are reviewed in Appendices A, B and $\mathrm{C}$.

\section{The addition formula for the $\tau$-function of the KP hierarchy}

Let

$$
[\alpha]=\left(\alpha, \frac{\alpha^{2}}{2}, \frac{\alpha^{3}}{3}, \ldots\right), \quad \xi(t, \lambda)=\sum_{n=1}^{\infty} t_{n} \lambda^{n}, \quad t=\left(t_{1}, t_{2}, t_{3}, \ldots\right) .
$$

The KP hierarchy is a system of equations for a function $\tau(t)[6,12,13]$ given by

$$
\oint e^{\xi\left(t^{\prime}-t, \lambda\right)} \tau\left(t^{\prime}-\left[\lambda^{-1}\right]\right) \tau\left(t+\left[\lambda^{-1}\right]\right) \frac{d \lambda}{2 \pi i}=0 .
$$

Here $\oint$ means a formal algebraic operator extracting the coefficient of $z^{-1}$ of Laurent series:

$$
\oint \frac{d z}{2 \pi i} \sum_{n=-\infty}^{\infty} a_{n} z^{n}=a_{-1} .
$$

Set $t=x+y, t^{\prime}=x-y$. Then (5) becomes

$$
\oint e^{-2 \xi(y, \lambda)} \tau\left(x-y-\left[\lambda^{-1}\right]\right) \tau\left(x+y+\left[\lambda^{-1}\right]\right) \frac{d \lambda}{2 \pi i}=0 .
$$

For an integer $m \geq 2$, set

$$
y=\frac{1}{2}\left(\sum_{i=1}^{m-1}\left[\beta_{i}\right]-\sum_{i=1}^{m+1}\left[\alpha_{i}\right]\right)
$$

in (6). Then it becomes

$$
\begin{aligned}
\oint \exp & \left(-\xi\left(\sum_{i=1}^{m-1}\left[\beta_{i}\right]-\sum_{i=1}^{m+1}\left[\alpha_{i}\right], \lambda\right)\right) \tau\left(x-\frac{1}{2}\left(\sum_{i=1}^{m-1}\left[\beta_{i}\right]-\sum_{i=1}^{m+1}\left[\alpha_{i}\right]\right)-\left[\lambda^{-1}\right]\right) \\
\times & \tau\left(x+\frac{1}{2}\left(\sum_{i=1}^{m-1}\left[\beta_{i}\right]-\sum_{i=1}^{m+1}\left[\alpha_{i}\right]\right)+\left[\lambda^{-1}\right]\right) \frac{d \lambda}{2 \pi i}=0 .
\end{aligned}
$$

By virtue of the identity

$$
\sum_{n=1}^{\infty} \frac{x^{n}}{n}=-\log (1-x)
$$

the exponential factor in (8) reduces to a rational function of $\lambda, \alpha_{i}, \beta_{i}$ as

$$
\exp \left(-\xi\left(\sum_{i=1}^{m-1}\left[\beta_{i}\right]-\sum_{i=1}^{m+1}\left[\alpha_{i}\right], \lambda\right)\right)=\frac{\prod_{i=1}^{m-1}\left(1-\beta_{i} \lambda\right)}{\prod_{i=1}^{m+1}\left(1-\alpha_{i} \lambda\right)} .
$$


Computing the integral by taking residues at $\lambda=\alpha_{i}^{-1}, 1 \leq i \leq m+1[15]$ and shifting the variable $x$ as

$$
x \rightarrow x+\frac{1}{2}\left(\sum_{i=1}^{m-1}\left[\beta_{i}\right]+\sum_{i=1}^{m+1}\left[\alpha_{i}\right]\right),
$$

we get the following addition formulae of the $\tau$-function [20]

$$
\sum_{i=1}^{m+1}(-1)^{i-1} \zeta\left(x ; \beta_{1}, \ldots, \beta_{m-1}, \alpha_{i}\right) \zeta\left(x ; \alpha_{1}, \ldots, \hat{\alpha}_{i}, \ldots, \alpha_{m+1}\right)=0, \quad m \geq 2,
$$

where

$$
\begin{aligned}
& \zeta\left(x ; \alpha_{1}, \ldots, \alpha_{n}\right)=\Delta\left(\alpha_{1}, \ldots, \alpha_{n}\right) \tau\left(x+\left[\alpha_{1}\right]+\cdots+\left[\alpha_{n}\right]\right), \\
& \Delta\left(\alpha_{1}, \ldots, \alpha_{n}\right)=\prod_{i<j}\left(\alpha_{i}-\alpha_{j}\right),
\end{aligned}
$$

and $\hat{\alpha}_{i}$ means that $\alpha_{i}$ should be removed.

Example 1. In the case of $m=2$, we have

$$
\begin{gathered}
\alpha_{12} \alpha_{34} \tau\left(x+\left[\alpha_{1}\right]+\left[\alpha_{2}\right]\right) \tau\left(x+\left[\alpha_{3}\right]+\left[\alpha_{4}\right]\right)-\alpha_{13} \alpha_{24} \tau\left(x+\left[\alpha_{1}\right]+\left[\alpha_{3}\right]\right) \tau\left(x+\left[\alpha_{2}\right]+\left[\alpha_{4}\right]\right) \\
+\alpha_{14} \alpha_{23} \tau\left(x+\left[\alpha_{1}\right]+\left[\alpha_{4}\right]\right) \tau\left(x+\left[\alpha_{2}\right]+\left[\alpha_{3}\right]\right)=0
\end{gathered}
$$

where $\alpha_{i j}=\alpha_{i}-\alpha_{j}$.

We call (10) 'the three term equation'. We have derived (10) from (5). The fact that the converse is true is proved by Takasaki and Takebe [23].

Theorem 1 ([23]). The three term equation (10) is equivalent to the KP hierarchy (5).

In [23] the theorem is proved by constructing the wave function of the KP-hierarchy. To do it the differential Fay identity, which is a certain limit of (10), is used. Here we give an alternative and direct proof of the theorem.

Proposition 1. The KP hierarchy (5) is equivalent to (9).

Proof. It is sufficient to prove that if (8) holds for any $m \geq 2$ and arbitrary $\alpha_{i} \neq 0, \beta_{i}$, then (6) holds. Set the left hand side of (6) to be $F(y)$. Expand $F(y)$ in $y$ as

$$
F(y)=\sum_{\gamma} F_{\gamma} y^{\gamma}, \quad y^{\gamma}=y_{1}^{\gamma_{1}} y_{2}^{\gamma_{2}} \cdots, \quad \gamma=\left(\gamma_{1}, \gamma_{2}, \ldots\right)
$$

We consider the case $\beta_{i}=0,1 \leq i \leq m-1$ in (7) and set

$$
y^{\prime}=\sum_{i=1}^{m+1}\left[\alpha_{i}\right]
$$

We prove $F_{\gamma}=0$ for any $\gamma$ if $F\left(-\frac{y^{\prime}}{2}\right)=0$ for any $m \geq 2$. We consider $m$ fixed. Let us define the weight of $y_{i}$ to be $i$ and wt $y^{\gamma}=\sum_{i=1}^{\infty} i \gamma_{i}$. Decompose $F$ according to weights as

$$
F=F^{(0)}+F^{(1)}+F^{(2)}+\cdots, \quad F^{(i)}=\sum_{\text {wt } y^{\gamma}=i} F_{\gamma} y^{\gamma}
$$


We substitute $-\frac{y^{\prime}}{2}$ to $y$ and get the homogeneous polynomial of degree $i$ of $\alpha_{1}, \ldots, \alpha_{m+1}$ :

$$
F^{(i)}\left(-\frac{y^{\prime}}{2}\right)=\sum_{\gamma_{1}+\cdots+\gamma_{m+1}=i} b_{\gamma_{1} \ldots \gamma_{m+1}}^{(i)} \alpha_{1}^{\gamma_{1}} \cdots \alpha_{m+1}^{\gamma_{m+1}} .
$$

Then $F\left(-\frac{y^{\prime}}{2}\right)=0$ is equivalent to $F^{(i)}\left(-\frac{y^{\prime}}{2}\right)=0$ for any $i$. Notice that $i y_{i}^{\prime}=\alpha_{1}^{i}+\cdots+\alpha_{m+1}^{i}$ is a power sum symmetric function. Therefore $y_{1}^{\prime}, \ldots, y_{m+1}^{\prime}$ are algebraically independent (see [14, (2.12)]). If $i \leq m+1$, then $F^{(i)}(y)$ is a polynomial at most of $y_{1}, \ldots, y_{m+1}$. Thus $F^{(i)}\left(-\frac{y^{\prime}}{2}\right)=0$ implies $F_{\gamma}=0$ for any $\gamma$ satisfying wt $\gamma=i$. Since $m$ is arbitrary we have $F_{\gamma}=0$ for any $\gamma$.

Remark 1. In the course of the proof we actually prove the equivalence between (5) and (9) with $\beta_{i}=0$ for any $i$ (of course, in that case we have firstly to divide $(9)$ by $\Delta\left(\beta_{1}, \ldots, \beta_{m-1}\right)$ ).

Proposition 2. The following formula follows from (10):

$$
\begin{aligned}
\frac{\tau\left(x+\sum_{i=1}^{m}\left[\beta_{i}\right]-\sum_{i=1}^{m}\left[\alpha_{i}\right]\right)}{\tau(x)} & \\
\quad= & \frac{\prod_{i, j=1}^{m}\left(\beta_{i}-\alpha_{j}\right)}{\prod_{i<j} \alpha_{i j} \beta_{j i}} \operatorname{det}\left(\frac{\tau\left(x+\left[\beta_{i}\right]-\left[\alpha_{j}\right]\right)}{\left(\beta_{i}-\alpha_{j}\right) \tau(x)}\right)_{1 \leq i, j \leq m}, \quad m \geq 2 .
\end{aligned}
$$

Proof. We change the names of $\alpha$ variables in (10) as $\left(\alpha_{3}, \alpha_{4}\right) \rightarrow\left(\beta_{1}, \beta_{2}\right)$ and shift $x$ to $x \rightarrow x-\left[\alpha_{1}\right]-\left[\alpha_{2}\right]$. After that we solve it in $\tau\left(x+\left[\beta_{1}\right]+\left[\beta_{2}\right]-\left[\alpha_{1}\right]-\left[\alpha_{2}\right]\right)$ we get $m=2$ case of (11).

Suppose that (11) holds in case of $m=k$ :

$$
\tau\left(x+\sum_{i=1}^{k}\left[\beta_{i}\right]-\sum_{i=1}^{k}\left[\alpha_{i}\right]\right)=\tau(x)^{-k+1} C_{k} \operatorname{det}\left(\frac{\tau\left(x+\left[\beta_{i}\right]-\left[\alpha_{j}\right]\right)}{\beta_{i}-\alpha_{j}}\right)_{1 \leq i, j \leq k},
$$

where

$$
C_{k}=\frac{\prod_{i, j=1}^{k}\left(\beta_{i}-\alpha_{j}\right)}{\prod_{i<j} \alpha_{i j} \beta_{j i}} .
$$

In (12) we shift the variable $x$ as

$$
x \rightarrow x+\left[\beta_{k+1}\right]-\left[\alpha_{k+1}\right] .
$$

Then

$$
\begin{gathered}
\tau\left(x+\sum_{i=1}^{k+1}\left[\beta_{i}\right]-\sum_{i=1}^{k+1}\left[\alpha_{i}\right]\right)=\tau\left(x+\left[\beta_{k+1}\right]-\left[\alpha_{k+1}\right]\right)^{-k+1} C_{k} \\
\times \operatorname{det}\left(\frac{\tau\left(x+\left[\beta_{i}\right]+\left[\beta_{k+1}\right]-\left[\alpha_{j}\right]-\left[\alpha_{k+1}\right]\right)}{\beta_{i}-\alpha_{j}}\right)_{1 \leq i, j \leq k} .
\end{gathered}
$$

By (11) with $m=2$,

$$
\tau\left(x+\left[\beta_{i}\right]+\left[\beta_{k+1}\right]-\left[\alpha_{j}\right]-\left[\alpha_{k+1}\right]\right)=\tau(x)^{-1} A_{i j} \cdot X_{i j},
$$


where

$$
\begin{aligned}
& A_{i j}=\frac{\left(\beta_{i}-\alpha_{j}\right)\left(\beta_{i}-\alpha_{k+1}\right)\left(\beta_{k+1}-\alpha_{j}\right)\left(\beta_{k+1}-\alpha_{k+1}\right)}{\left(\alpha_{j}-\alpha_{k+1}\right)\left(\beta_{k+1}-\beta_{i}\right)}, \\
& X_{i j}=\operatorname{det}\left(\begin{array}{cc}
\frac{\tau\left(x+\left[\beta_{i}\right]-\left[\alpha_{j}\right]\right)}{\beta_{i}-\alpha_{j}} & \frac{\tau\left(x+\left[\beta_{i}\right]-\left[\alpha_{k+1}\right]\right)}{\beta_{i}-\alpha_{k+1}} \\
\frac{\tau\left(x+\left[\beta_{k+1}\right]-\left[\alpha_{j}\right]\right)}{\beta_{k+1}-\alpha_{j}} & \frac{\tau\left(x+\left[\beta_{k+1}\right]-\left[\alpha_{k+1}\right]\right)}{\beta_{k+1}-\alpha_{k+1}}
\end{array}\right) .
\end{aligned}
$$

By substituting (14) to the determinant in the right hand side of (13), we have

$$
\begin{aligned}
\operatorname{det}( & \left.\frac{\tau\left(x+\left[\beta_{i}\right]+\left[\beta_{k+1}\right]-\left[\alpha_{j}\right]-\left[\alpha_{k+1}\right]\right)}{\beta_{i}-\alpha_{j}}\right)_{1 \leq i, j \leq k} \\
& =\left(\frac{\beta_{k+1}-\alpha_{k+1}}{\tau(x)}\right)^{k} \prod_{i=1}^{k} \frac{\left(\beta_{k+1}-\alpha_{i}\right)\left(\beta_{i}-\alpha_{k+1}\right)}{\left(\alpha_{i}-\alpha_{k+1}\right)\left(\beta_{k+1}-\beta_{i}\right)} \operatorname{det}\left(X_{i j}\right)_{1 \leq i, j \leq k} .
\end{aligned}
$$

Using Sylvester's theorem (Appendix B),

$$
\operatorname{det}\left(X_{i j}\right)_{1 \leq i, j \leq k}=\left(\frac{\tau\left(x+\left[\beta_{k+1}\right]-\left[\alpha_{k+1}\right]\right)}{\beta_{k+1}-\alpha_{k+1}}\right)^{k-1} \operatorname{det}\left(\frac{\tau\left(x+\left[\beta_{i}\right]-\left[\alpha_{j}\right]\right)}{\beta_{i}-\alpha_{j}}\right)_{1 \leq i, j \leq k+1} .
$$

Substituting (16) and (15) into (13), we get the case of $m=k+1$ of (11).

Let us consider an $N \times m$ matrix $\mathcal{A}=\left(a_{i j}\right)_{1 \leq i \leq N, 1 \leq j \leq m}$ with $N \geq m$ and set, for $1 \leq$ $l_{1}, \ldots, l_{m} \leq N$

$$
A\left(l_{1}, \ldots, l_{m}\right)=\operatorname{det}\left(a_{l_{i}, j}\right)_{1 \leq i, j \leq m} .
$$

For any $1 \leq k_{1}, \ldots, k_{m-1}, l_{1}, \ldots, l_{m+1} \leq N$ these determinants satisfy Plücker's relations:

$$
\sum_{i=1}^{m+1}(-1)^{i-1} A\left(k_{1}, \ldots, k_{m-1}, l_{i}\right) A\left(l_{1}, \ldots, \hat{l}_{i}, \ldots, l_{m+1}\right)=0 .
$$

Proposition 3. The Plücker's relations for the determinant of the right hand side of (11) give the addition formulae (9).

Proof. Let $m$ be fixed. Consider the $\infty \times m$ matrix $\mathcal{A}=\left(a_{i j}\right)$ with

$$
a_{i j}=\frac{\tau\left(x+\left[\beta_{i}\right]-\left[\alpha_{j}\right]\right)}{\left(\beta_{i}-\alpha_{j}\right) \tau(x)} .
$$

Then $A\left(k_{1}, \ldots, k_{m-1}, l_{i}\right)$ and $A\left(l_{1}, \ldots, \hat{l}_{i}, \ldots, l_{m+1}\right)$ can be expressed by $2 m$ point functions by (11). We substitute them to (17) and shift the variable $x$ as

$$
x \rightarrow x+\sum_{s=1}^{m}\left[\alpha_{s}\right]
$$

Then we get the additional formulae (9) by renaming the variables as $\left(\beta_{k_{1}}, \ldots, \beta_{k_{m-1}}\right) \rightarrow$ $\left(\beta_{1}, \ldots, \beta_{m-1}\right),\left(\beta_{l_{1}}, \ldots, \beta_{l_{m+1}}\right) \rightarrow\left(\alpha_{1}, \ldots, \alpha_{m+1}\right)$.

Proof of Theorem 1. By Propositions 1, 2 and 3, we have (5) from (10). Thus Theorem 1 is proved. 


\section{3 mKP hierarchy}

Let $\tau_{l}(t)(l \in \mathbb{Z})$ be $\tau$-functions of the modified KP (mKP) hierarchy. We use the same notation as that for KP hierarchy $([\alpha], \xi(t, \lambda)$, etc.).

The mKP hierarchy is given by the bilinear equation $[6,12]$ of the form

$$
\oint e^{\xi\left(t^{\prime}-t, \lambda\right)} \lambda^{l-l^{\prime}} \tau_{l}\left(t^{\prime}-\left[\lambda^{-1}\right]\right) \tau_{l^{\prime}}\left(t+\left[\lambda^{-1}\right]\right) \frac{d \lambda}{2 \pi i}=0, \quad l \geq l^{\prime} .
$$

Set $t=x+y, t^{\prime}=x-y$. Then (18) becomes

$$
\oint e^{-2 \xi(y, \lambda)} \lambda^{l-l^{\prime}} \tau_{l}\left(x-y-\left[\lambda^{-1}\right]\right) \tau_{l^{\prime}}\left(x+y+\left[\lambda^{-1}\right]\right) \frac{d \lambda}{2 \pi i}=0, \quad l \geq l^{\prime} .
$$

Let $l-l^{\prime}=k \geq 0$. Set

$$
y=\frac{1}{2}\left(\sum_{i=1}^{m-2}\left[\beta_{i}\right]-\sum_{i=1}^{m+k}\left[\alpha_{i}\right]\right) .
$$

Then the exponential factor in (19) reduces to a rational function of $\lambda, \alpha_{i}, \beta_{i}$ as in the $\mathrm{KP}$ case:

$$
\exp \left(-\xi\left(\sum_{i=1}^{m-2}\left[\beta_{i}\right]-\sum_{i=1}^{m+k}\left[\alpha_{i}\right], \lambda\right)\right)=\frac{\prod_{i=1}^{m-2}\left(1-\beta_{i} \lambda\right)}{\prod_{i=1}^{m+k}\left(1-\alpha_{i} \lambda\right)} .
$$

Computing the integral by taking residues at $\lambda=\alpha_{i}^{-1}, 1 \leq i \leq m+k$ and shifting the variable $x$ as

$$
x \rightarrow x+\frac{1}{2}\left(\sum_{i=1}^{m-2}\left[\beta_{i}\right]+\sum_{i=1}^{m+k}\left[\alpha_{i}\right]\right),
$$

we have the following addition formulae of the mKP hierarchy:

$$
\begin{aligned}
& \sum_{i=1}^{m+k}(-1)^{i-1} \zeta_{l}\left(x ; \beta_{1}, \ldots, \beta_{m-2}, \alpha_{i}\right) \zeta_{l+k}\left(x ; \alpha_{1}, \ldots, \hat{\alpha}_{i}, \ldots, \alpha_{m+k}\right)=0, \\
& \quad l \in \mathbb{Z}, \quad k \geq 0, \quad m \geq 2,
\end{aligned}
$$

where

$$
\zeta_{l}\left(x ; \alpha_{1}, \ldots, \alpha_{n}\right)=\Delta\left(\alpha_{1}, \ldots, \alpha_{n}\right) \tau_{l}\left(x+\sum_{i=1}^{n}\left[\alpha_{i}\right]\right) .
$$

Example 2. The case $l-l^{\prime}=1$ and $m=2$ of $(20)$ is

$$
\begin{aligned}
& \alpha_{23} \tau_{l}\left(x+\left[\alpha_{1}\right]\right) \tau_{l+1}\left(x+\left[\alpha_{2}\right]+\left[\alpha_{3}\right]\right)-\alpha_{13} \tau_{l}\left(x+\left[\alpha_{2}\right]\right) \tau_{l+1}\left(x+\left[\alpha_{1}\right]+\left[\alpha_{3}\right]\right) \\
& +\alpha_{12} \tau_{l}\left(x+\left[\alpha_{3}\right]\right) \tau_{l+1}\left(x+\left[\alpha_{1}\right]+\left[\alpha_{2}\right]\right)=0 .
\end{aligned}
$$

We call (21) 'the three term equation of the mKP hierarchy'.

The following theorem is proved in [17].

Theorem 2 ([17]). The three term equation (21) is equivalent to the mKP hierarchy (18). 
We give an another proof which is similar to that of the KP hierarchy. The following proposition can be proved as in the KP-case.

Proposition 4. The mKP hierarchy (18) is equivalent to (20).

Proposition 5. The following formula follows from (21) for $n \geq 2$ :

$$
\begin{aligned}
& \frac{\tau_{l+1}\left(x+\sum_{i=1}^{n}\left[\alpha_{i}\right]-\sum_{i=1}^{n-1}\left[\beta_{i}\right]\right)}{\tau_{l}(x)} \\
&=C \operatorname{det}\left(\begin{array}{cccc}
\frac{\tau_{l}\left(x+\left[\alpha_{1}\right]-\left[\beta_{1}\right]\right)}{\left(\alpha_{1}-\beta_{1}\right) \tau_{l}(x)} & \cdots & \frac{\tau_{l}\left(x+\left[\alpha_{1}\right]-\left[\beta_{n-1}\right]\right)}{\left(\alpha_{1}-\beta_{n-1}\right) \tau_{l}(x)} & \frac{\tau_{l+1}\left(x+\left[\alpha_{1}\right]\right)}{\tau_{l}(x)} \\
\frac{\tau_{l}\left(x+\left[\alpha_{2}\right]-\left[\beta_{1}\right]\right)}{\left(\alpha_{2}-\beta_{1}\right) \tau_{l}(x)} & \cdots & \frac{\tau_{l}\left(x+\left[\alpha_{2}\right]-\left[\beta_{n-1}\right]\right)}{\left(\alpha_{2}-\beta_{n-1}\right) \tau_{l}(x)} & \frac{\tau_{l+1}\left(x+\left[\alpha_{2}\right]\right)}{\tau_{l}(x)} \\
\vdots & \ddots & \vdots \\
\frac{\tau_{l}\left(x+\left[\alpha_{n}\right]-\left[\beta_{1}\right]\right)}{\left(\alpha_{n}-\beta_{1}\right) \tau_{l}(x)} & \cdots & \frac{\tau_{l}\left(x+\left[\alpha_{n}\right]-\left[\beta_{n-1}\right]\right)}{\left(\alpha_{n}-\beta_{n-1}\right) \tau_{l}(x)} & \frac{\tau_{l+1}\left(x+\left[\alpha_{n}\right]\right)}{\tau_{l}(x)}
\end{array}\right),
\end{aligned}
$$

where

$$
C=C\left(l,\left\{\alpha_{i}\right\},\left\{\beta_{i}\right\}\right)=\frac{\prod_{i=1}^{n} \prod_{j=1}^{n-1}\left(\alpha_{i}-\beta_{j}\right)}{\left(\prod_{i<j}^{n-1} \beta_{i j}\right)\left(\prod_{i>j}^{n} \alpha_{i j}\right)} .
$$

Proof. The proof is similar to that of Proposition 2. Therefore we leave details to readers.

Proposition 6. The Plücker's relations for the determinant of the right hand side of (22) give (20) with $k=1$.

Proof. For $m \geq 1$ consider the $m \times 2 m$ matrix $\mathcal{A}=\left(a_{i j}\right)_{1 \leq i \leq m, 1 \leq j \leq 2 m}$ given by

$$
a_{i j}=\frac{\tau_{l}\left(x+\left[\alpha_{i}\right]-\left[\beta_{j}\right]\right)}{\alpha_{i}-\beta_{j}}, \quad 1 \leq j \leq 2 m-1, \quad a_{i, 2 m}=\tau_{l+1}\left(x+\left[\alpha_{i}\right]\right) .
$$

For $1 \leq r_{1}, \ldots, r_{m} \leq 2 m$ we set

$$
A\left(r_{1}, \ldots, r_{m}\right)=\operatorname{det}\left(a_{i, r_{j}}\right)_{1 \leq i, j \leq m}
$$

Then the Plücker's relation gives $k=1$ case of (20) by Propositions 2 and 5 .

By Propositions 5 and 6, equation (20) with $k=1$ and arbitrary $m \geq 2$ follows from (21). The next lemma shows that (20) with $k \geq 2$ and $m \geq 2$ also follows from (21). The fact that (20) with $k=0$ follows from (21) is proved in [17]. We generalize the arguments in [17] for $k \geq 2$.

Lemma 1. Equation (20) follows from (21).

Proof. We prove the lemma by induction on $k$. Suppose that equation (20) is valid for $k$ and any $m \geq 2$. Shift the variable $x$ as

$$
x \rightarrow x-\sum_{j=1}^{m+k-1}\left[\alpha_{j}\right],
$$


and multiply the resulting equation by $\tau_{l+k+1}\left(x+\left[\alpha_{m+k+1}\right]\right)$. Then we get

$$
\begin{aligned}
& \sum_{i=1}^{m+k-1} A_{i} \tau_{l}\left(x-\sum_{j \neq i}^{m+k-1}\left[\alpha_{j}\right]+\sum_{j=1}^{m-2}\left[\beta_{j}\right]\right) \tau_{l+k}\left(x+\left[\alpha_{m+k}\right]-\left[\alpha_{i}\right]\right) \tau_{l+k+1}\left(x+\left[\alpha_{m+k+1}\right]\right) \\
& +A_{m+k} \tau_{l}\left(x+\left[\alpha_{m+k}\right]+\sum_{j=1}^{m-2}\left[\beta_{j}\right]-\sum_{j=1}^{m+k-1}\left[\alpha_{j}\right]\right) \tau_{l+k}(x) \tau_{l+k+1}\left(x+\left[\alpha_{m+k}\right]\right)=0
\end{aligned}
$$

where

$$
A_{i}=(-1)^{i-1} \Delta\left(\beta_{1}, \ldots, \beta_{m-2}, \alpha_{i}\right) \Delta\left(\alpha_{1}, \ldots, \hat{\alpha}_{i}, \ldots, \alpha_{m+k}\right) .
$$

In (21) with $l$ being replaced by $l+k$, make a shift $x \rightarrow x-\left[\alpha_{3}\right]$ and change the label of $\alpha$ as $\left(\alpha_{1}, \alpha_{2}, \alpha_{3}\right) \rightarrow\left(\alpha_{m+k}, \alpha_{m+k+1}, \alpha_{i}\right), 1 \leq i \leq m+k-1$. Then we get

$$
\begin{aligned}
\tau_{l+k}(x & \left.+\left[\alpha_{m+k}\right]-\left[\alpha_{i}\right]\right) \tau_{l+k+1}\left(x+\left[\alpha_{m+k+1}\right]\right) \\
= & \frac{\alpha_{m+k, i}}{\alpha_{m+k+1, i}} \tau_{l+k}\left(x+\left[\alpha_{m+k+1}\right]-\left[\alpha_{i}\right]\right) \tau_{l+k+1}\left(x+\left[\alpha_{m+k}\right]\right) \\
& \quad-\frac{\alpha_{m+k, m+k+1}}{\alpha_{m+k+1, i}} \tau_{l+k}(x) \tau_{l+k+1}\left(x+\left[\alpha_{m+k}\right]+\left[\alpha_{m+k+1}\right]-\left[\alpha_{i}\right]\right) .
\end{aligned}
$$

Substituting (24) to the summands of (23) and shifting $x$ as $x \rightarrow x+\sum_{j=1}^{m+k-1}\left[\alpha_{j}\right]$, then we get

$$
\begin{aligned}
\tau_{l+k+1} & \left(x+\sum_{j=1}^{m+k}\left[\alpha_{j}\right]\right) \sum_{i=1}^{m+k-1} A_{i} \frac{\alpha_{m+k, i}}{\alpha_{m+k+1, i}} \tau_{l}\left(x+\left[\alpha_{i}\right]+\sum_{j=1}^{m-2}\left[\beta_{j}\right]\right) \\
& \times \tau_{l+k}\left(x+\sum_{j \neq i}^{m+k-1}\left[\alpha_{j}\right]+\left[\alpha_{m+k+1}\right]\right)+\tau_{l+k}\left(x+\sum_{j=1}^{m+k-1}\left[\alpha_{j}\right]\right) \\
& \times\left\{\sum_{i=1}^{m+k-1} A_{i} \frac{\alpha_{m+k, m+k+1}}{\alpha_{i, m+k+1}} \tau_{l}\left(x+\left[\alpha_{i}\right]+\sum_{j=1}^{m-2}\left[\beta_{j}\right]\right) \tau_{l+k+1}\left(x+\sum_{j \neq i}^{m+k+1}\left[\alpha_{j}\right]\right)\right. \\
& \left.+A_{m+k} \tau_{l}\left(x+\left[\alpha_{m+k}\right]+\sum_{j=1}^{m-2}\left[\beta_{j}\right]\right) \tau_{l+k+1}\left(x+\sum_{j \neq m+k}^{m+k+1}\left[\alpha_{j}\right]\right)\right\}=0 .
\end{aligned}
$$

We write (20) in the form

$$
\begin{aligned}
& \sum_{i=1}^{m+k-1} A_{i} \tau_{l}\left(x+\left[\alpha_{i}\right]+\sum_{j=1}^{m-2}\left[\beta_{j}\right]\right) \tau_{l+k}\left(x+\sum_{j \neq i}^{m+k}\left[\alpha_{j}\right]\right) \\
& \quad=-A_{m+k} \tau_{l}\left(x+\left[\alpha_{m+k}\right]+\sum_{j=1}^{m-2}\left[\beta_{j}\right]\right) \tau_{l+k}\left(x+\sum_{j=1}^{m+k-1}\left[\alpha_{j}\right]\right) .
\end{aligned}
$$

Change $\alpha_{m+k}$ to $\alpha_{m+k+1}$ in (26). Notice that $A_{i}, i<m+k$, changes to

$$
\begin{aligned}
(-1)^{i-1} \Delta\left(\beta_{1}, \ldots, \beta_{m-2}, \alpha_{i}\right) \Delta\left(\alpha_{1}, \ldots, \hat{\alpha}_{i}, \ldots, \alpha_{m+k-1}, \alpha_{m+k+1}\right) \\
\quad=\prod_{j=1}^{m+k-1} \frac{\alpha_{j, m+k+1}}{\alpha_{j, m+k}} \cdot A_{i} \cdot \frac{\alpha_{i, m+k}}{\alpha_{i, m+k+1}}
\end{aligned}
$$


and $A_{m+k}$ changes to

$$
(-1)^{m+k} \Delta\left(\beta_{1}, \ldots, \beta_{m-2}, \alpha_{m+k+1}\right) \Delta\left(\alpha_{1}, \ldots, \alpha_{m+k-1}\right) .
$$

Then we can rewrite the first term of $(25)$ as

$$
\begin{aligned}
\tau_{l+k+1} & \left(x+\sum_{j=1}^{m+k}\left[\alpha_{j}\right]\right) \sum_{i=1}^{m+k-1} A_{i} \frac{\alpha_{i, m+k}}{\alpha_{i, m+k+1}} \tau_{l}\left(x+\left[\alpha_{i}\right]+\sum_{j=1}^{m-2}\left[\beta_{j}\right]\right) \\
\times & \tau_{l+k}\left(x+\sum_{j \neq i}^{m+k-1}\left[\alpha_{j}\right]+\left[\alpha_{m+k+1}\right]\right)=\tau_{l+k+1}\left(x+\sum_{j=1}^{m+k}\left[\alpha_{j}\right]\right) B_{m+k+1} \\
& \times \tau_{l}\left(x+\left[\alpha_{m+k+1}\right]+\sum_{j=1}^{m-2}\left[\beta_{j}\right]\right) \tau_{l+k}\left(x+\sum_{j=1}^{m+k-1}\left[\alpha_{j}\right]\right)
\end{aligned}
$$

where

$$
B_{m+k+1}=(-1)^{m+k+1} \frac{\Delta\left(\alpha_{m+k+1}, \beta_{1}, \ldots, \beta_{m-2}\right) \Delta\left(\alpha_{1}, \ldots, \alpha_{m+k}\right)}{\prod_{j=1}^{m+k-1} \alpha_{j, m+k+1}} .
$$

Substitute (27) to (25) and divide the resulting equation by $\tau_{l+k}\left(x+\sum_{j=1}^{m+k-1}\left[\alpha_{j}\right]\right)$. We, then, multiply it by $\prod_{j=1}^{m+k-1} \alpha_{j, m+k+1}$ and get the case of $k+1$ of $(20)$.

\section{BKP hierarchy}

Let $\tau(t)$ be the $\tau$-function of the BKP hierarchy. In this case, the time variable is $t=$ $\left(t_{1}, t_{3}, t_{5}, \ldots\right)$. We set

$$
[\alpha]_{\mathrm{o}}=\left(\alpha, \frac{\alpha^{3}}{3}, \frac{\alpha^{5}}{5}, \ldots\right), \quad \tilde{\xi}(t, \lambda)=\sum_{n=1}^{\infty} t_{2 n-1} \lambda^{2 n-1} .
$$

The BKP hierarchy $[6,12]$ is defined by

$$
\oint e^{\tilde{\xi}\left(t-t^{\prime}, \lambda\right)} \tau\left(t-2\left[\lambda^{-1}\right]_{\mathrm{o}}\right) \tau\left(t^{\prime}+2\left[\lambda^{-1}\right]_{\mathrm{o}}\right) \frac{d \lambda}{2 \pi i \lambda}=\tau(t) \tau\left(t^{\prime}\right) .
$$

Set $t=x-y, t^{\prime}=x+y$. We get

$$
\oint e^{-2 \tilde{\xi}(y, \lambda)} \tau\left(x-y-2\left[\lambda^{-1}\right]_{\mathrm{o}}\right) \tau\left(x+y+2\left[\lambda^{-1}\right]_{\mathrm{o}}\right) \frac{d \lambda}{2 \pi i \lambda}=\tau(x-y) \tau(x+y) .
$$

Set

$$
y=\sum_{i=1}^{n}\left[\alpha_{i}\right]_{\mathrm{o}}
$$

in (29). Then we have

$$
\oint e^{-2 \tilde{\xi}\left(\sum_{i=1}^{n}\left[\alpha_{i}\right]_{\mathrm{o}}, \lambda\right)} \tau\left(x-\sum_{i=1}^{n}\left[\alpha_{i}\right]_{\mathrm{o}}-2\left[\lambda^{-1}\right]_{\mathrm{o}}\right) \tau\left(x+\sum_{i=1}^{n}\left[\alpha_{i}\right]_{\mathrm{o}}+2\left[\lambda^{-1}\right]_{\mathrm{o}}\right) \frac{d \lambda}{2 \pi i \lambda}
$$




$$
=\tau\left(x-\sum_{i=1}^{n}\left[\alpha_{i}\right]_{\mathrm{o}}\right) \tau\left(x+\sum_{i=1}^{n}\left[\alpha_{i}\right]_{\mathrm{o}}\right) .
$$

By decomposing $-2 \sum_{n=1}^{\infty} t_{2 n-1} \lambda^{2 n-1}$ as

$$
-2 \sum_{n=1}^{\infty} t_{2 n-1} \lambda^{2 n-1}=-\sum_{n=1}^{\infty} t_{n} \lambda^{n}+\sum_{n=1}^{\infty} t_{n}(-\lambda)^{n},
$$

we get

$$
\exp \left(-2 \tilde{\xi}\left(\sum_{i=1}^{n}\left[\alpha_{i}\right]_{\mathrm{o}}, \lambda\right)\right)=\prod_{i=1}^{n} \frac{1-\alpha_{i} \lambda}{1+\alpha_{i} \lambda} .
$$

Computing the integral by taking residues as before, shifting $x$ as $x+\sum_{i=1}^{n}\left[\alpha_{i}\right]_{\mathrm{o}}$ and dividing by $\tau(x)^{2}$ we have

$$
\begin{aligned}
& \sum_{i=1}^{n}(-1)^{i-1} \frac{\tau\left(x+2\left[\alpha_{i}\right]_{\mathrm{o}}\right)}{\tau(x)} A_{1 \ldots \hat{i} \ldots n}^{-1} \frac{\tau\left(x+2 \sum_{l=1, l \neq i}^{n}\left[\alpha_{l}\right]_{\mathrm{o}}\right)}{\tau(x)} \\
& -A_{1 \ldots n}^{-1} \frac{\tau\left(x+2 \sum_{l=1}^{n}\left[\alpha_{l}\right]_{\mathrm{o}}\right)}{\tau(x)}=0, \quad n \text { odd }, \\
& \sum_{i=1}^{n-1}(-1)^{i-1} \frac{\alpha_{i, n} \frac{\tau\left(x+2\left[\alpha_{i}\right]_{\mathrm{o}}+2\left[\alpha_{n}\right]_{\mathrm{o}}\right)}{\tau(x)} A_{1 \ldots \hat{i} \ldots n-1}^{-1} \frac{\tau\left(x+2 \sum_{l=1, l \neq i}^{n-1}\left[\alpha_{l}\right]_{\mathrm{o}}\right)}{\tau(x)}}{-A_{1 \ldots n}^{-1} \frac{\tau\left(x+2 \sum_{l=1}^{n}\left[\alpha_{l}\right]_{\mathrm{o}}\right)}{\tau(x)}=0, \quad n \text { even. }}
\end{aligned}
$$

Here $A_{1 \ldots n}$ is defined by

$$
A_{1 \ldots n}=\prod_{i<j}^{n} \frac{\tilde{\alpha}_{i j}}{\alpha_{i j}}, \quad \tilde{\alpha}_{i j}=\alpha_{i}+\alpha_{j}, \quad \alpha_{i j}=\alpha_{i}-\alpha_{j} .
$$

Example 3. The case $n=3$ of (30) becomes

$$
\begin{aligned}
\frac{\tau\left(x+2 \sum_{i=1}^{3}\left[\alpha_{i}\right]_{\mathrm{o}}\right)}{\tau(x)}= & A_{123}\left\{\frac{\tau\left(x+2\left[\alpha_{1}\right]_{\mathrm{o}}\right)}{\tau(x)} \frac{\alpha_{23}}{\tilde{\alpha}_{23}} \frac{\tau\left(x+2\left[\alpha_{2}\right]_{\mathrm{o}}+2\left[\alpha_{3}\right]_{\mathrm{o}}\right)}{\tau(x)}\right. \\
& -\frac{\tau\left(x+2\left[\alpha_{2}\right]_{\mathrm{o}}\right)}{\tau(x)} \frac{\alpha_{13}}{\tilde{\alpha}_{13}} \frac{\tau\left(x+2\left[\alpha_{1}\right]_{\mathrm{o}}+2\left[\alpha_{3}\right]_{\mathrm{o}}\right)}{\tau(x)} \\
& \left.+\frac{\tau\left(x+2\left[\alpha_{3}\right]_{\mathrm{o}}\right)}{\tau(x)} \frac{\alpha_{12}}{\tilde{\alpha}_{12}} \frac{\tau\left(x+2\left[\alpha_{1}\right]_{\mathrm{o}}+2\left[\alpha_{2}\right]_{\mathrm{o}}\right)}{\tau(x)}\right\} .
\end{aligned}
$$

We call equation (32) 'the four term equation of the BKP hierarchy'. 
Example 4. The case of $n=4$ of (31) is

$$
\begin{aligned}
\frac{\tau\left(x+2 \sum_{i=1}^{4}\left[\alpha_{i}\right]_{\mathrm{o}}\right)}{\tau(x)}= & A_{1234}\left\{\frac{\alpha_{14}}{\tilde{\alpha}_{14}} \frac{\tau\left(x+2\left[\alpha_{1}\right]_{\mathrm{o}}+2\left[\alpha_{4}\right]_{\mathrm{o}}\right)}{\tau(x)} \frac{\alpha_{23}}{\tilde{\alpha}_{23}} \frac{\tau\left(x+2\left[\alpha_{2}\right]_{\mathrm{o}}+2\left[\alpha_{3}\right]_{\mathrm{o}}\right)}{\tau(x)}\right. \\
& -\frac{\alpha_{24}}{\tilde{\alpha}_{24}} \frac{\tau\left(x+2\left[\alpha_{2}\right]_{\mathrm{o}}+2\left[\alpha_{4}\right]_{\mathrm{o}}\right)}{\tau(x)} \frac{\alpha_{13}}{\tilde{\alpha}_{13}} \frac{\tau\left(x+2\left[\alpha_{1}\right]_{\mathrm{o}}+2\left[\alpha_{3}\right]_{\mathrm{o}}\right)}{\tau(x)} \\
& \left.+\frac{\alpha_{34}}{\tilde{\alpha}_{34}} \frac{\tau\left(x+2\left[\alpha_{3}\right]_{\mathrm{o}}+2\left[\alpha_{4}\right]_{\mathrm{o}}\right)}{\tau(x)} \frac{\alpha_{12}}{\tilde{\alpha}_{12}} \frac{\tau\left(x+2\left[\alpha_{1}\right]_{\mathrm{o}}+2\left[\alpha_{2}\right]_{\mathrm{o}}\right)}{\tau(x)}\right\} .
\end{aligned}
$$

As is proved in Proposition 8, equation (33) can be derived from equation (32).

Theorem 3. The four term equation (32) is equivalent to the BKP hierarchy (28).

We prove this theorem in a similar way to the case of the KP hierarchy.

In order to prove the theorem, we use Pfaffians. The definition and notation of Pfaffians are reviewed in Appendix A.

Let us define the components of Pfaffians by

$$
(0, j)=\frac{\tau\left(x+2\left[\alpha_{j}\right]_{\mathrm{o}}\right)}{\tau(x)}, \quad(i, j)=\frac{\alpha_{i j}}{\tilde{\alpha}_{i j}} \frac{\tau\left(x+2\left[\alpha_{i}\right]_{\mathrm{o}}+2\left[\alpha_{j}\right]_{\mathrm{o}}\right)}{\tau(x)} .
$$

Then it is possible to rewrite (32) and (33) as

$$
\begin{aligned}
& \frac{\tau\left(x+2 \sum_{i=1}^{3}\left[\alpha_{i}\right]_{\mathrm{o}}\right)}{\tau(x)}=A_{123}(0,1,2,3), \\
& \frac{\tau\left(x+2 \sum_{i=1}^{4}\left[\alpha_{i}\right]_{\mathrm{o}}\right)}{\tau(x)}=A_{1234}(1,2,3,4),
\end{aligned}
$$

respectively.

The following proposition can be proved in a similar manner to Proposition 1.

Proposition 7. The BKP hierarchy (28) is equivalent to (30) and (31).

Proposition 8. The following equations are implied by (32):

$$
\begin{aligned}
& \frac{\tau\left(x+2 \sum_{i=1}^{n}\left[\alpha_{i}\right]_{\mathrm{o}}\right)}{\tau(x)}=A_{1 \ldots n}(0,1,2, \ldots, n), \quad n \geq 3, \text { odd }, \\
& \frac{\tau\left(x+2 \sum_{i=1}^{n}\left[\alpha_{i}\right]_{\mathrm{o}}\right)}{\tau(x)}=A_{1 \ldots n}(1,2, \ldots, n), \quad n \geq 4, \text { even } .
\end{aligned}
$$

Proof. First we prove that (32) implies (33). Shift $x$ in (32) as $x \rightarrow x+2\left[\alpha_{4}\right]_{\mathrm{o}}$ :

$$
\begin{aligned}
& \frac{\tau\left(x+2 \sum_{i=1}^{4}\left[\alpha_{i}\right]_{\mathrm{o}}\right)}{\tau\left(x+2\left[\alpha_{4}\right]_{\mathrm{o}}\right)} \\
& \quad=A_{123} \frac{\tau(x)^{2}}{\tau\left(x+2\left[\alpha_{4}\right]_{\mathrm{o}}\right)^{2}}\left\{\frac{\tau\left(x+2\left[\alpha_{1}\right]_{\mathrm{o}}+2\left[\alpha_{4}\right]_{\mathrm{o}}\right)}{\tau(x)} \frac{\alpha_{23}}{\tilde{\alpha}_{23}} \frac{\tau\left(x+2\left[\alpha_{2}\right]_{\mathrm{o}}+2\left[\alpha_{3}\right]_{\mathrm{o}}+2\left[\alpha_{4}\right]_{\mathrm{o}}\right)}{\tau(x)}\right.
\end{aligned}
$$




$$
\begin{aligned}
& -\frac{\tau\left(x+2\left[\alpha_{2}\right]_{\mathrm{o}}+2\left[\alpha_{4}\right]_{\mathrm{o}}\right)}{\tau(x)} \frac{\alpha_{13}}{\tilde{\alpha}_{13}} \frac{\tau\left(x+2\left[\alpha_{1}\right]_{\mathrm{o}}+2\left[\alpha_{3}\right]_{\mathrm{o}}+2\left[\alpha_{4}\right]_{\mathrm{o}}\right)}{\tau(x)} \\
& \left.+\frac{\tau\left(x+2\left[\alpha_{3}\right]_{\mathrm{o}}+2\left[\alpha_{4}\right]_{\mathrm{o}}\right)}{\tau(x)} \frac{\alpha_{12}}{\tilde{\alpha}_{12}} \frac{\tau\left(x+2\left[\alpha_{1}\right]_{\mathrm{o}}+2\left[\alpha_{2}\right]_{\mathrm{o}}+2\left[\alpha_{4}\right]_{\mathrm{o}}\right)}{\tau(x)}\right\} .
\end{aligned}
$$

Use (34) to rewrite $\tau\left(x+2\left[\alpha_{i_{1}}\right]_{\mathrm{o}}+2\left[\alpha_{i_{2}}\right]_{\mathrm{o}}+2\left[\alpha_{i_{3}}\right]_{\mathrm{o}}\right)$ in (38). Then we get (35). We prove (36) by induction on $n$. The case $n=3$ is obvious. Suppose that (36) holds in case of $n$ :

$$
\frac{\tau\left(x+2 \sum_{i=1}^{n}\left[\alpha_{i}\right]_{\mathrm{o}}\right)}{\tau(x)}=A_{1 \ldots n}(0,1,2, \ldots, n)=A_{1 \ldots n} \operatorname{Pf} \mathcal{A}
$$

where $\mathcal{A}=\left(a_{i j}\right)_{0 \leq i, j \leq n}$ is a skew-symmetric matrix,

$$
a_{i j}= \begin{cases}\frac{\tau\left(x+2\left[\alpha_{j}\right]_{\mathrm{o}}\right)}{\tau(x)}, & i=0, \\ \frac{\alpha_{i j}}{\tilde{\alpha}_{i j}} \frac{\tau\left(x+2\left[\alpha_{i}\right]_{\mathrm{o}}+2\left[\alpha_{j}\right]_{\mathrm{o}}\right)}{\tau(x)}, & i \neq 0, \quad i<j .\end{cases}
$$

In (39) we shift $x$ as

$$
x \rightarrow x+2\left[\alpha_{n+1}\right]_{\mathrm{o}}+2\left[\alpha_{n+2}\right]_{\mathrm{o}} .
$$

Then we have, using (34) and (35),

$$
\frac{\tau\left(x+2 \sum_{i=1}^{n+2}\left[\alpha_{i}\right]_{\mathrm{o}}\right)}{\tau(x)}=A_{1 \ldots n+2}(n+1, n+2)^{-\frac{n-1}{2}} \operatorname{Pf} \mathcal{B}
$$

where $\mathcal{B}=\left(b_{i j}\right)_{0 \leq i<j \leq n}$ is a skew-symmetric matrix given by

$$
b_{i j}=(n+1, n+2, i, j), \quad i<j .
$$

By the analogue of the Sylvester' theorem for Pfaffians (Appendix B), we have

$$
\operatorname{Pf}((1,2, \ldots, 2 r, i, j))_{2 r+1 \leq i<j \leq 2 m}=(1,2, \ldots, 2 r)^{m-r-1}(1,2, \ldots, 2 m) .
$$

Consider the case $r=1$ and $2 m=n+3$ of this formula:

$$
\operatorname{Pf}((n+1, n+2, i, j))_{0 \leq i<j \leq n}=(n+1, n+2)^{\frac{n-1}{2}}(n+1, n+2,0, \ldots, n) .
$$

Substituting (41) to (40), we get

$$
\frac{\tau\left(x+2 \sum_{i=1}^{n+2}\left[\alpha_{i}\right]_{\mathrm{o}}\right)}{\tau(x)}=A_{1 \ldots n+2}(0,1, \ldots, n+2) .
$$

The case of even $n$ is similarly proved.

Proposition 9. The Plücker's relations for Pfaffians of the right hand side of (36) and (37) give the addition formulae (30) and (31) respectively.

Proof. Using the Plücker's relations (43) and (44) for Pfaffians given in Appendix C, the proposition can easily be checked by direct calculations. 


\section{A Pfaffians}

Let $\mathcal{A}=\left(a_{i j}\right)_{1 \leq i, j \leq 2 m}$ be a skew-symmetric matrix. Then the Pfaffian $\operatorname{Pf} \mathcal{A}$ is defined by

$$
\operatorname{det} \mathcal{A}=(\operatorname{Pf} \mathcal{A})^{2}, \quad \operatorname{Pf} \mathcal{A}=a_{12} a_{34} \cdots a_{2 m-1,2 m}+\cdots .
$$

Following [10] we denote $\operatorname{Pf} \mathcal{A}$ by $(1,2,3, \ldots, 2 m)$ :

$$
\operatorname{Pf} \mathcal{A}=(1,2,3, \ldots, 2 m) \text {. }
$$

It is directly defined by

$$
(1,2,3, \ldots, 2 m)=\sum \operatorname{sgn}\left(i_{1}, \ldots, i_{2 m}\right) \cdot\left(i_{1}, i_{2}\right)\left(i_{3}, i_{4}\right) \cdots\left(i_{2 m-1}, i_{2 m}\right), \quad(i, j)=a_{i j},
$$

where the sum is over all permutations of $(1, \ldots, 2 m)$ such that

$$
i_{1}<i_{3}<\cdots<i_{2 m-1}, \quad i_{1}<i_{2}, \ldots, i_{2 m-1}<i_{2 m},
$$

and $\operatorname{sgn}\left(i_{1}, \ldots, i_{2 m}\right)$ is the signature of the permutation $\left(i_{1}, \ldots, i_{2 m}\right)$. The Pfaffian can be expanded as

$$
(1,2,3, \ldots, 2 m)=\sum_{j=2}^{2 m}(-1)^{j}(1, j)(2,3, \ldots, \hat{j}, \ldots, 2 m) .
$$

For example, in the case of $m=2$,

$$
(1,2,3,4)=(1,2)(3,4)-(1,3)(2,4)+(1,4)(2,3)
$$

\section{B Sylvester's theorem for determinants and Pfaffians}

The following theorem is known as Sylvester's theorem.

Theorem 4. Let $r \leq m, \mathcal{A}=\left(a_{i j}\right)_{1 \leq i, j \leq m}$ and $\mathcal{A}_{r}=\left(a_{i j}\right)_{1 \leq i, j \leq r}$. Set

$$
\mathcal{B}=\left(b_{i j}\right)_{r+1 \leq i, j \leq m}, \quad b_{i j}=\operatorname{det}\left(\begin{array}{cccc}
a_{11} & \ldots & a_{1 r} & a_{1 j} \\
\vdots & \ddots & \vdots & \vdots \\
a_{r 1} & \ldots & a_{r r} & a_{r j} \\
a_{i 1} & \ldots & a_{i r} & a_{i j}
\end{array}\right) \text {. }
$$

Then we get

$$
\operatorname{det} \mathcal{B}=\left(\operatorname{det} \mathcal{A}_{r}\right)^{m-r-1} \operatorname{det} \mathcal{A} .
$$

Let $\mathcal{A}=\left(a_{i j}\right)_{1 \leq i, j \leq 2 m}$ be a skew-symmetric matrix and set $(i, j)=a_{i j}$. For $r \leq m$ let $\mathcal{P}=\left(p_{i j}\right)_{2 r+1 \leq i, j \leq 2 m}, p_{i j}=(1,2, \ldots, 2 r, i, j)$ and $I_{r}=\{1,2, \ldots, 2 r\}$. In general, for a subset

$I \subset\{1,2, \ldots, 2 m\}$ we set $\mathcal{A}(I)=\left(a_{i j}\right)_{i, j \in I}$ and for $i<j, k<l$ we denote by $\mathcal{A}_{k l}^{i j}$ be the square matrix of degree $2(m-1)$ which is obtained from $\mathcal{A}$ by removing $i$-th and $j$-th rows, $k$-th and $l$-th columns.

Theorem 5 ([9]). For $r \leq m$ the following identity holds:

$$
\operatorname{Pf} \mathcal{P}=\left(\operatorname{Pf} \mathcal{A}\left(I_{r}\right)\right)^{m-r-1} \operatorname{Pf} \mathcal{A}
$$




\section{The Plücker relation for Pfaffians}

There exist analogues of the Plücker's relations for Pfaffians [18]. They are given by

$$
\begin{aligned}
& \sum_{l=1}^{L}(-1)^{l}\left(i_{1}, \ldots, i_{K}, j_{l}\right)\left(j_{1}, \ldots, \hat{j}_{l}, \ldots, j_{L}\right) \\
& \quad+\sum_{k=1}^{K}(-1)^{k}\left(i_{1}, \ldots, \hat{i}_{k}, \ldots, i_{K}\right)\left(j_{1}, \ldots, j_{L}, i_{k}\right)=0
\end{aligned}
$$

where $K$ and $L$ are odd. We understand that $(\varnothing)=1$.

For $n$ odd, taking $K=1, L=n, i_{1}=0$ and $j_{1}, \ldots, j_{n} \neq 0$ in (42), we get

$$
\sum_{l=1}^{n}(-1)^{l-1}\left(0, j_{l}\right)\left(j_{1}, \ldots, \hat{j}_{l}, \ldots, j_{n}\right)-\left(0, j_{1}, \ldots, j_{n}\right)=0 .
$$

For $n$ even, setting $K=1, L=n-1$ and $i_{1} \neq 0$ in (42), we have

$$
\sum_{l=1}^{n-1}(-1)^{l-1}\left(i_{1}, j_{l}\right)\left(j_{1}, \ldots, \hat{j}_{l}, \ldots, j_{n-1}\right)-\left(i_{1}, j_{1}, \ldots, j_{n-1}\right)=0 .
$$

\section{Acknowledgements}

I would like to thank Masatoshi Noumi and Takashi Takebe for permitting me to see the manuscript [17] prior to its publication. I also thank Kanehisa Takasaki and Takashi Takebe for insightful comments and their interests in the present work. I also thank Yasuhiro Ohta and Soichi Okada for important comments about Pfaffians and informing me about reference [9]. Finally I am deeply grateful to Atsushi Nakayashiki for much advice. This research is supported by JSPS Grant-in-Aid for Scientific Research (B) No. 23340037.

\section{References}

[1] Bobenko A.I., Suris Yu.B., Discrete differential geometry. Integrable structure, Graduate Studies in Mathematics, Vol. 98, Amer. Math. Soc., Providence, RI, 2008.

[2] Buchstaber V.M., Enolski V.Z., Leykin D.V., Kleinian functions, hyperelliptic Jacobians and applications, Rev. Math and Math. Phys. 10 (1997), no. 2, 1-125, solv-int/9603005.

[3] Date E., Jimbo M., Miwa T., Method for generating discrete soliton equations. I, J. Phys. Soc. Japan 51 (1982), 4116-4124.

[4] Date E., Jimbo M., Miwa T., Method for generating discrete soliton equations. II, J. Phys. Soc. Japan 51 (1982), 4125-4131.

[5] Date E., Jimbo M., Miwa T., Method for generating discrete soliton equations. V, J. Phys. Soc. Japan 52 (1982), 766-771.

[6] Date E., Kashiwara M., Jimbo M., Miwa T., Transformation groups for soliton equations, in Nonlinear Integrable Systems - Classical Theory and Quantum Theory (Kyoto, 1981), World Sci. Publishing, Singapore, 1983, 39-119.

[7] Eilbeck J.C., Enolski V.Z., Gibbons J., Sigma, tau and Abelian functions of algebraic curves, J. Phys. A: Math. Theor. 43 (2010), 455216, 20 pages, arXiv:1006.5219.

[8] Fay J.D., Theta functions on Riemann surfaces, Lecture Notes in Mathematics, Vol. 352, Springer-Verlag, Berlin, 1973.

[9] Hirota R., Generalizations of determinant identities by Pfaffian, in Mathematical Theories and Applications of Nonlinear Waves and Nonlinear Dynamics, Research Institute for Applied Mechanics, Kyushu University, 2004, 148-156. 
[10] Hirota R., The direct method in soliton theory, Cambridge Tracts in Mathematics, Vol. 155, Cambridge University Press, Cambridge, 2004.

[11] Inoguchi J., Kajiwara K., Matsuura N., Ohta Y., Explicit solutions to the semi-discrete modified KdV equation and motion of discrete plane curves, arXiv:1108.1328.

[12] Jimbo M., Miwa T., Solitons and infinite-dimensional Lie algebras, Publ. Res. Inst. Math. Sci. 19 (1983), 943-1001.

[13] Miwa T., Jimbo M., Date E., Solitons. Differential equations, symmetries and infinite-dimensional algebras, Cambridge Tracts in Mathematics, Vol. 135, Cambridge University Press, Cambridge, 2000.

[14] Macdonald I.G., Symmetric functions and Hall polynomials, 2nd ed., Oxford Mathematical Monographs, The Clarendon Press, Oxford University Press, New York, 1995.

[15] Miwa T., On Hirota's difference equations, Proc. Japan Acad. Ser. A Math. Sci. 58 (1982), 9-12.

[16] Nakayashiki A., Sigma function as a tau function, Int. Math. Res. Not. 2010 (2010), no. 3, 373-394, arXiv:0904.0846.

[17] Noumi M., Takebe T., Algebraic analysis of integrable hierarchies, in preparation.

[18] Ohta Y., Bilinear theory of solitons with Pfaffian labels, Sūrikaisekikenkyūsho Kōkyūroku (1993), no. 822, $197-205$.

[19] Raina A.K., Fay's trisecant identity and conformal field theory, Comm. Math. Phys. 122 (1989), 625-641.

[20] Sato M., Sato Y., Soliton equations as dynamical systems on infinite dimensional Grassmann manifold, in Nonlinear PDE in Applied Science, North-Holland Math. Stud., Vol. 81, Editors H. Fujita, P. Lax, G. Strang, Tokyo, 1982, 259-271.

[21] Takasaki K., Differential Fay identities and auxiliary linear problem of integrable hierarchies, in Exploring New Structures and Natural Constructions in Mathematical Physics, Adv. Stud. Pure Math., Vol. 61, Math. Soc. Japan, Tokyo, 2011, 387-441, arXiv:0710.5356.

[22] Takasaki K., Dispersionless Hirota equations of two-component BKP hierarchy, SIGMA 2 (2006), 057, 22 pages, nlin.SI/0604003.

[23] Takasaki K., Takebe T., Integrable hierarchies and dispersionless limit, Rev. Math. Phys. 7 (1995), 743-808, hep-th/9405096.

[24] Teo L.P., Fay-like identities of the Toda lattice hierarchy and its dispersionless limit, Rev. Math. Phys. 18 (2006), 1055-1073, nlin.SI/0606059. 